\title{
Robotic Repair of Congenital Paraesophageal Hiatal Hernia
}

\author{
Daniel A. DeUgarte, MD, ${ }^{1}$ Ronald B. Hirschl, ${ }^{2}$ and James D. Geiger ${ }^{2}$
}

\begin{abstract}
Congenital paraesophageal hiatal hernias are rare and can be associated with gastric incarceration, volvulus, mucosal ulceration, and anemia. Primary repair of the hernia and fundoplication are recommended. In this paper, we report a case of a 3-year-old child with abdominal pain who was noted to have a paraesophageal hiatal hernia with partial gastric volvulus. A $5 \mathrm{~mm}$ robot platform was utilized to facilitate hernia sac dissection, hiatal repair, and fundoplication.
\end{abstract}

\section{Introduction}

CongenitAl OR PRIMARY paraesophageal hiatal hernias are rare and they commonly present clinically with incarceration of the stomach. ${ }^{1-3}$ Antenatal diagnosis has been reported, but the average age of presentation is 23 months. ${ }^{2,4}$ Surgical repair is advocated because of the risk of gastric volvulus, strangulation, mucosal ulceration, anemia, and associated chest infections. Most surgeons recommend primary repair without a reinforcing patch. A fundoplication is advocated to prevent an otherwise high incidence of reflux. ${ }^{2}$ Minimally invasive repair of intrathoracic stomach has been described. ${ }^{5}$ In this paper, we report the robotic-assisted repair of a paraesophageal hiatal hernia with partial gastric volvulus.

\section{Case}

A 3-year-old $15 \mathrm{~kg}$ girl with pectus excavatum presented with a history of intermittent bouts of severe abdominal pain and feeling "that she needed to burp." A chest radiograph was performed and demonstrated an intrathoracic air-fluid level (Fig. 1). An upper gastrointestinal contrast study demonstrated a paraesophageal hiatal hernia with partial gastric volvulus (Fig. 2). A CT scan performed at the referring hospital delineated the pectus deformity and intrathoracic stomach (Fig. 3). The patient was referred for surgical intervention, and a minimally-invasive repair was planned.

A $5 \mathrm{~mm}$ first-generation da Vinci ${ }^{\circledR}$ robotic platform (Intuitive Surgical, Inc., Sunnyvale, CA) was utilized. Four $5 \mathrm{~mm}$ trocars were utilized, and a stab incision in the epigastric region was utilized for insertion of a liver retractor (Fig. 4). The umbilical port was used to introduce a two-dimensional 5 $\mathrm{mm}$ da Vinci ${ }^{\circledR}$ camera. Right and left ports were used for 5 mm da Vinci ${ }^{\circledR}$ instruments. A fourth $5 \mathrm{~mm}$ trocar in the left abdomen was used by an assistant to help provide traction on the stomach during the dissection.

A large portion of the stomach was herniated into the chest through the esophageal hiatus. A hernia sac was noted and required extensive dissection with care to preserve the blood supply to the stomach and to prevent injury to the vagus nerve. A partial volvulus was noted, and the stomach was derotated. Once the esophageal hiatus was clearly delineated, silk sutures were used to approximate the defect posteriorly. In order to prevent undue angulation of the esophagus, additional sutures were placed anteriorly to close the hiatal defect. A slightly foreshortened esophagus was sutured to the adjacent diaphragm. A floppy Nissen wrap was then fashioned. The patient was discharged home a few days following the operation. In follow-up, she was noted to have resolution of her symptoms without dysphagia.

\section{Discussion}

Minimally-invasive techniques have been applied to treat gastroesophageal reflux disease, paraesophageal hernias, achalasia, and benign and malignant esophageal disease. ${ }^{6}$ Robot-assisted minimally-invasive surgery has the added benefit of enhanced visualization with three-dimensional (3D) optics and improved maneuverability with articulating instruments. Furthermore, the robot offers the ability to scale motion and provides the ergonomic advantage of having the surgeon seated comfortably at a console. This technology can be particularly helpful when working in narrow spaces such as the pelvis during prostatectomy and in the mediastinum during transhiatal esophagectomy. In adults undergoing transhiatal esophagectomy, the robot platform has been demonstrated to minimize blood loss, reduce postoperative

${ }^{1}$ Division of Pediatric Surgery, UCLA School of Medicine, Los Angeles, California.

${ }^{2}$ Division of Pediatric Surgery, University of Michigan, Ann Arbor, Michigan. 


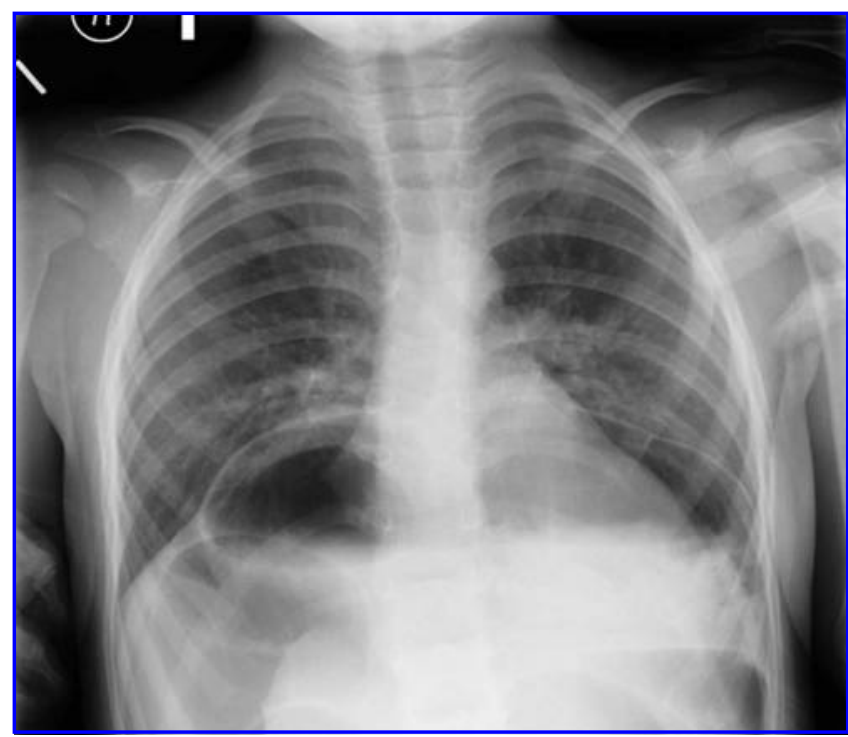

FIG. 1. Chest radiograph demonstrates an intrathoracic airfluid level.

pain, and decrease hospital stay without compromising oncological principles. ${ }^{7,8}$ However, the operative duration of robotic procedures appears to be increased because of added setup and procedural time.

Utilization of the robotic platform in pediatric surgery has been limited by instrument size and cost. ${ }^{9}$ By utilizing the 5 $\mathrm{mm}$ camera, we did not have the advent of 3D visualization, which is currently available only with the $10 \mathrm{~mm}$ camera. The smallest instruments available $(5 \mathrm{~mm})$ articulate using a series of mechanical links that move in succession resulting in "snake-like" movements. ${ }^{10}$ Unfortunately, these added movements in combination with the remote-center trocar distance robot requirements minimize the working area. ${ }^{10}$ These limitations make robotic procedures in neonates especially challenging.

The "snake-like" $5 \mathrm{~mm}$ instruments were not as "intuitive" as their $8 \mathrm{~mm}$ counterparts, but they did facilitate hernia sac dissection and suture approximation of the crura. We were also pleased with the Schertel grasper, which worked effectively to grasp and reduce the stomach without undue tissue trauma. The concern for excess force and tissue damage was of concern given the absence of normal haptic feedback with the robot. This proved not to be an issue in our experience. Many pediatric surgeons elect not to use the robot platform because many of the operations can be performed with smaller incision using $3 \mathrm{~mm}$ instruments. Nonetheless, there is still a potential role for the robot in cases where the added articulation and instrument maneuverability may facilitate the operation and improve the outcomes.

From a resource utilization standpoint, the operation had a prolonged setup and procedural time. Furthermore, a cosurgeon was needed at the bedside to help retract and reduce the herniated stomach and to help exchange the $5 \mathrm{~mm}$ instruments. Proper room-setup, patient positioning, and trocar placement in the setting of a dedicated team can help to streamline robot-assisted procedures. ${ }^{9}$

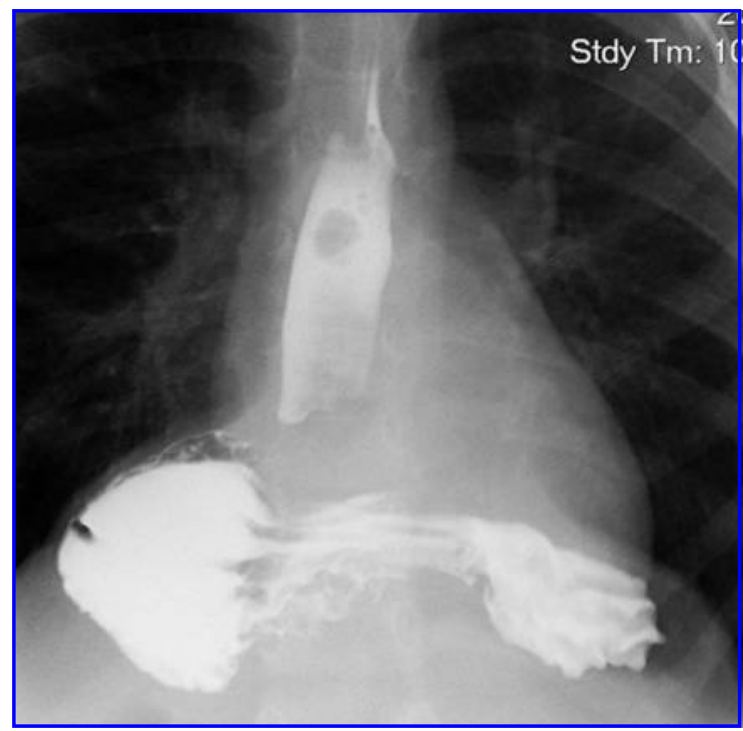

FIG. 2. Upper gastrointestinal contrast study demonstrates an intrathoracic stomach with partial gastric volvulus.

\section{Conclusion}

Surgical repair of congenital or primary paraesophageal hiatal hernia is advocated because of the risks of gastric volvulus, strangulation, mucosal ulceration, and anemia. Most surgeons recommend primary repair and fundoplication. The hernia-sac dissection and suture approximation of the crura was facilitated by the $5 \mathrm{~mm}$ robotic instruments. However, the potential benefits of the robot must be taken into context when similar results can technically be achieved by a skilled laparoscopic surgeon using $3 \mathrm{~mm}$ instruments. Furthermore, instrument size and cost must be taken into consideration when deciding to use the robot platform.

\section{Disclosure Statement}

No competing financial interests exist.

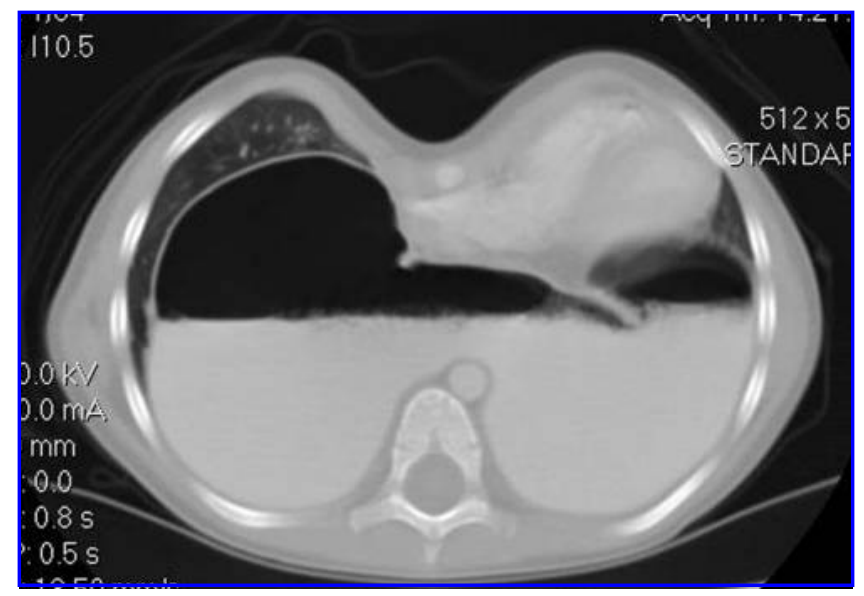

FIG. 3. Computed tomography scan delineates the pectus deformity and confirms the intrathoracic stomach. 


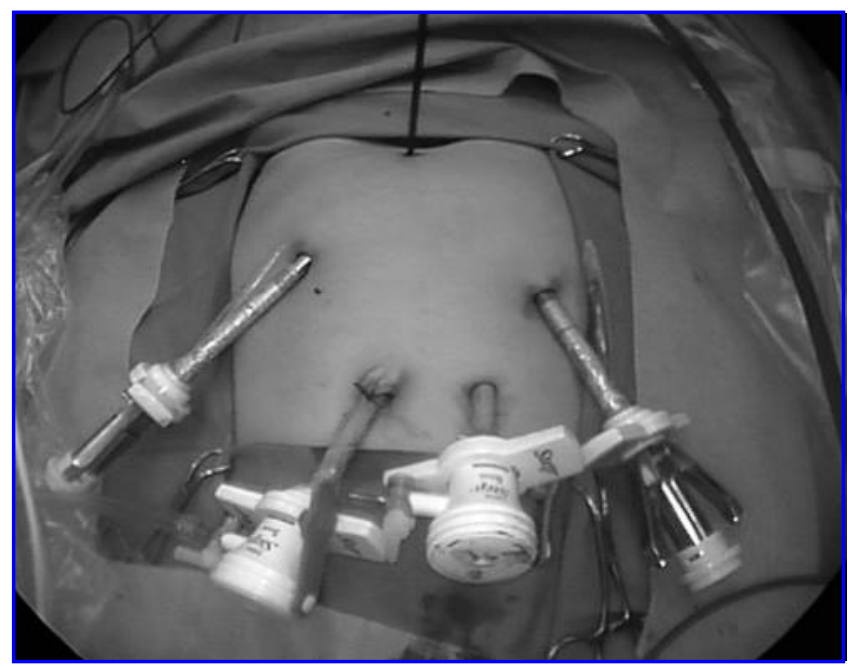

FIG. 4. Trocar placement. An umbilical trocar was used for the camera. A stab incision was used in the epigastrium for retraction of the liver. $5 \mathrm{~mm}$ da Vinci robotic trocars were placed laterally. An additional trocar was placed in the midleft abdomen for the assistant to help retract the stomach.

\section{References}

1. Rawat J, Rashid KA, Sinha SK, et al. Congenital paraesophageal hiatal hernia with intrathoracic gastric volvulus in an infant: a case report with radiographic sequence. Pediatr Surg Int 2008;24:467-470.

2. Karpelowsky, JS, Wieselthaler N, Rode H. Primary paraesophageal hernia in children. J Pediatr Surg 2006;41: 1588-1593.

3. Imamoglu M, Cay A, Kosucu P, et al. Congenital paraesophageal hiatal hernia: pitfalls in the diagnosis and treatment. J Pediatr Surg 2005;40:1128-1133.
4. Al-Assiri A, Wiseman N, Bunge M. Prenatal diagnosis of intrathoracic stomach (gastric herniation). I Pediatr Surg 40:E15-17, 20055.

5. Yagi M, Nose K, Yamauchi K, et al. Laparoscopic intervention for intrathoracic stomach in infants. Surg Endosc 2003; 17:1636-1639.

6. Hanly EJ, Talamini MA. Robotic abdominal surgery. Am J Surg 2004;188:19S-26S.

7. Braghetto I, Csendes A, Cardemil G, Burdiles P, Korn O, Valladares $\mathrm{H}$. Open transthoracic or transhiatal esophagectomy versus minimally invasive esophagectomy in terms of morbidity, mortality, and survival. Surg Endosc 2006; 20:1681-1686.

8. Galvani C, Gorodner MV, Moser F, Jacobsen G, Chretien C, Espat NJ, Donahue P, Horgan S. Robotically assisted laparoscopic transhiatal esophagectomy. Surg Endosc 2008;22: 188-195.

9. Meehan, J, Sandler A. Pediatric robotic surgery: A single-institutional review of the first 100 consecutive cases. Surg Endosc 2008;22:177-182.

10. Meehan J, Sandler A. Robotic repair of a bochdalek congenital diaphragmatic hernia in a small neonate: robotic advantages and limitations. I Pediatr Surg 2007;42:17571760.
Address reprint requests to: Daniel A. DeUgarte, MD

Division of Pediatric Surgery UCLA School of Medicine 10833 Le Conte Avenue Box 957098

Los Angeles, CA 90095-7098

E-mail: deugarte@post.harvard.edu 